\title{
Maximum Number of Actual Assessments
}

National Cancer Institute

\section{Source}

National Cancer Institute. Maximum Number of Actual Assessments. NCI Thesaurus.

Code C117303.

The upper limit in number of acutal assessments during a planned period of time. 